\title{
Robust Control for Discrete-Time Singular Marovian Jump Systems with Partly Unknown Transition Rates
}

\author{
Yuhong Liu ${ }^{1,}$, , Hui Li ${ }^{1}$, Qishui Zhong ${ }^{1}$, Shouming Zhong ${ }^{2,3}$ \\ ${ }^{1}$ School of Aeronautics and Astronautics, University of Electronic Science and Technology of China, Chengdu, China \\ ${ }^{2}$ School of Mathematical Sciences, University of Electronic Science and Technology of China, Chengdu, China \\ ${ }^{3}$ Key Laboratory for Neuroinformation of Ministry of Education, University of Electronic Science and Technology of China, Chengdu, China
}

Email address:

yhliu1210@163.com (Yuhong Liu), kelly.li@126.com (Hui Li), zhongqs@uestc.edu.cn (Qishui Zhong)

${ }^{*}$ Corresponding author

\section{To cite this article:}

Yuhong Liu, Hui Li, Qishui Zhong, Shouming Zhong. Robust Control for Discrete-Time Singular Marovian Jump Systems with Partly Unknown Transition Rates. Science Journal of Applied Mathematics and Statistics. Vol. 4, No. 5, 2016, pp. 217-224.

doi: 10.11648/j.sjams.20160405.14

Received: August 24, 2016; Accepted: September 12, 2016; Published: September 23, 2016

\begin{abstract}
This study investigates the problem of robust control for a class of discrete-time singular Marovian jump systems with partly unknown transition rates. Linear matrix inequality (LMI)-based sufficient conditions for the stochastic stability and robust control are developed. Then, a static output feedback controller and a robust static output feedback controller are designed to make sure the closed-loop systems are piecewise regular, causal and stochastically stable. Finally, numerical examples are presented to demonstrate the effectiveness and advantages of the theoretical results.
\end{abstract}

Keywords: Robust Control, Partly Unknown Transition Rates, Singular Markovian Jump Systems

\section{Introduction}

Singular systems, which are also referred to as descriptor systems, differential-algebraic systems, generalized state-space systems and semistate-space systems, provide convenient and natural representations in the description of circuits system [1], power systems [2], economic system [3], singular biological systems [4] and so on. There are some results have been reported on stability analysis and control design for the singular systems [5-10], where not only the asymptotic stability, but the system regularity and free-impulse/causal problems are considered for a class for singular systems.

In recent years, the study of Markovian jump systems problem has attracted considerable attentions of many researchers. Markovian jump systems are an important class of stochastic systems, which are popular in modelling many practical systems that may experience random abrupt changes in their structures and parameters [11-24]. More recently, there are some initial studies on singular systems with Markovian switching, for the discrete-time case, see [11], the problems of stability, state feedback control and static output feedback control for a class of discrete-time singular hybrid systems were investigated, and a new sufficient and necessary condition guaranteeing the system to be regular, causal and stochastically stable was proposed in terms of a set of coupled strict LMIs. But, the results developed in these references require the critical assumption on the complete knowledge of the transition probabilities in the jump process, see [11-25]. [26] proposes the less conservative stabilization conditions for MJSs with incomplete knowledge of transition probabilities and input saturation. The delay-dependent stability problem for neutral Markovian jump systems with generally unknown transition rates was investigated in [27]. In [27], each transition rate is completely unknown or only its estimate value is known. Based on the study of expectations of the stochastic cross-terms containing the Ito ${ }^{\wedge}$ integral, a new stability criterion is derived in terms of linear matrix inequalities.

This paper considers the robust control of discrete-time uncertain singular Markovian jump system systems with partially unknown transition probabilities of this paper are as follows: (1). Design of a static output feedback controller for the systems with partially unknown transition probabilities by LMIs. (2). The technique of design of a static output feedback 
controller for the systems with partially unknown transition probabilities is extended to the uncertain systems by LMIs. (3). It is shown that the solution of the matrix inequalities in this paper can be more easily to obtain, which use two matrices $W_{1}$ and $W_{2}$ instead of two scalars in [11] to solve the output feedback controller by example 1 .

Notation: The superscripts $T$ and (-1) stand for matrix transposition and matrix inverse, respectively; $R^{n}$ denotes the $n$-dimensional Euclidean space; $Z$ denotes the set of non-negative integer numbers; the notation $X>Y(X \geq Y)$, where $\mathrm{X}, \mathrm{Y}$ are symmetric matrices, means that $\mathrm{X}-\mathrm{Y}$ positive definite (positive semidefinite). $*$ denotes the term that is induced by symmetry. $(\Omega, F, \mathrm{P})$ denotes a complete probability space, in which $\Omega$ is the sample space, $F$ is the $\sigma$ algebra of subsets of the sample space, and $\mathrm{P}$ is the probability measure on $F$. Matrices, if their dimensions are not explicitly stated, are assumed to have appropriate dimensions for algebraic operations. For simplicity, sometimes $A_{i}, B_{i}, \Delta A_{i}, \Delta B_{i}$ and $K_{i}$ are used to denote $A\left(r_{k}\right), B\left(r_{k}\right), C\left(r_{k}\right), \Delta A\left(r_{k}\right), \Delta B\left(r_{k}\right)$, and $K\left(r_{k}\right)$, respectively.

\section{Problem Formulation and Preliminaries}

Consider the following discrete-time singular Markovian jump systems with an interval time-varying delay in the state, defined on a complete probability space $(\Omega, F, \mathrm{P})$

$$
\left\{\begin{aligned}
& E x(k+1)=\left(A\left(r_{k}\right)+\Delta A\left(r_{k}\right)\right) x(k)+\left(B_{u}\left(r_{k}\right)\right. \\
&+\Delta B\left(r_{k}\right) u(k), \\
& y(k)=C\left(r_{k}\right) x(k),
\end{aligned}\right.
$$

where $x(k) \in R^{n}$ is the system state, $y(k) \in R^{p}$ is the output vector, $u(k) \in R^{m}$ is the input vector, $A\left(r_{t}\right) \in R^{n \times n}$, $B_{u}\left(r_{t}\right) \in R^{n \times m}$, and $C\left(r_{t}\right) \in R^{p \times m}$ are known real constant matrices with appropriate dimensions, and $C\left(r_{t}\right) \in R^{p \times n}$ are assumed to be of full row rank. The matrix $E \in R^{n \times n}$ may be singular, with; The matrices $\Delta A\left(r_{k}\right)$ and $\Delta B\left(r_{k}\right)$ are unknown matrices representing parameter uncertainties, and are assumed to be of the form

$$
\left[\Delta A_{i} \Delta B_{i}\right]=D_{i} F_{i}(k)\left[M_{1 i} M_{2 i}\right],
$$

where $D_{i}, M_{1 i}$ and $M_{2 i}$ are known real constant matrices, and are unknown matrix functions satisfying

$$
F_{i}(k)^{T} F_{i}(k) \leq I
$$

$\left\{r_{k}, k \geq 0\right\}$ is the jumping process. $\left\{r_{k}\right\}$ is a discrete time homogeneous Markovian process with right discrete trajectories which takes values in a finite set $l=\{1,2, \ldots, N\}$, with transition probability matrix $\pi=\left[\pi_{i j}\right]_{N \times N}$, and $\pi_{i j} \geq 0$ is defined as

$$
\pi_{i j}=\operatorname{Pr}\left\{r_{k+1}=j \mid r_{k}=i\right\}
$$

Where $\sum_{j=1}^{N} \pi_{i, \mathrm{j}}=1$, and the Markovian process transition probability matrix $\pi$ is defined by

$$
\pi=\left(\begin{array}{cccc}
\pi_{11} & \pi_{12} & \cdots & \pi_{1 N} \\
\pi_{21} & \pi_{22} & \cdots & \pi_{2 N} \\
\vdots & \vdots & \ddots & \vdots \\
\pi_{N 1} & \pi_{N 2} & \cdots & \pi_{N N}
\end{array}\right),
$$

In addition, the transition probabilities of the jumping process are considered to be all known and partially accessed in this paper, some elements, for the systems eq.(1) with 4 operation modes, the transition probability matrix $\pi$ may be expressed three cases as following:

$$
\pi=\left(\begin{array}{cccc}
\pi_{11} & \pi_{12} & \pi_{13} & \pi_{14} \\
? & ? & ? & \pi_{24} \\
\pi_{31} & ? & \pi_{33} & ? \\
? & ? & ? & ?
\end{array}\right)
$$

Where "?" represents the inaccessible elements. For notational clarity, $\forall i \in l$ we denote $l=l_{k}^{i} \cup l_{u k}^{i}$ where

$$
l_{k}^{i}=\left\{j: \pi_{i j} \text { is known }\right\}, \quad l_{u k}^{i}=\left\{j: \pi_{i j} \text { is unknown }\right\},
$$

Moreover, if $l_{k}^{i} \neq \varnothing$, it is further described as $l_{k}^{i}=\left\{k_{1}^{i}, k_{2}^{i}, \ldots, k_{m}^{i}\right\}, \forall 1 \leq m \leq N$, where $k_{m}^{i} \in N^{+}$represents the $m$ th known element with index in the $i$ th row of matrix $\pi$. Because $\Sigma_{j=1}^{N} \pi_{i, j}=1$ and $\sum_{j_{1} \in l_{k}^{i}} \pi_{i, j_{1}}+\Sigma_{j_{2} \in l_{u k}^{i}} \pi_{i, j_{2}}=1$, we denote

$$
h_{i}=\sum_{j_{2} \in l_{u k}^{i}} \pi_{i, j_{2}}=1-\sum_{j_{1} \in l_{k}^{i}} \pi_{i, j_{1}}
$$

Where $j_{1} \in l_{k}^{i}, j_{2} \in l_{u k}^{i}$

Definition 1

(1). The discrete-time singular Markovian jump systems in eq. (1) with $\Delta A\left(r_{k}\right), \Delta B\left(r_{k}\right), u(k)=0$ are said to be regular if, for each $i \in l, \operatorname{det}\left(z E-A_{i}\right)$ is not identically zero.

(2). The discrete-time singular Markovian jump systems eq. (1) with $\Delta A\left(r_{k}\right), \Delta B\left(r_{k}\right), u(k)=0$ are said to be regular if, for each $i \in l$,

$$
\operatorname{deg}\left(\operatorname{det}\left(z E-A_{i}\right)\right)=\operatorname{rank}(E) .
$$

(3). The discrete-time singular Markovian jump systems eq. 
(1) with $\Delta A\left(r_{k}\right), \Delta B\left(r_{k}\right), u(k)=0$ are said to be stochastically admissible if for any $x_{0} \in R^{n}$ and $r_{0} \in l$, there exists a scalar $M\left(x_{0}, r_{0}\right)$ such that

$$
E\left\{\sum_{k=0}^{\infty}\|x(k)\|^{2} \mid x_{0}, r_{0}\right\}<M\left(x_{0}, r_{0}\right),
$$

Where $x\left(k, x_{0}, r_{0}\right)$ denotes the solution to the systems eq. (1) at time $k$ under the initial conditions $x_{0}$ and $r_{0}$.

(4). The discrete-time singular Markovian jump systems eq. (1) with $\Delta A\left(r_{k}\right), \Delta B\left(r_{k}\right), u(k)=0$ are said to be stochastically admissible if they are regular, causal and stochastically stable.

Define $R \in R^{n \times n}$ as the matrix with the properties of $E^{T} R^{T}=0$ and which are used in all the subsequent lemmas and theorems.

Lemma 1: Let $L_{i}$ be nonsingular matrices with appropriate dimensions, for $i \in l$. Then, the inequalities

$$
A_{i}^{T}\left(\sum_{j=1}^{N} \pi_{i, j} P_{j}-R^{T} \Phi R\right) A_{i}-E^{T} P_{i} E<0,
$$

Hold if for

$$
\left[\begin{array}{cc}
\Pi_{i} & A_{i}^{T} L_{i}-L_{i}^{T} \\
L_{i}^{T} A_{i}-L_{i} & \sum_{j \in l}^{N} \pi_{i, j} P_{j}-L_{i}-L_{i}^{T}
\end{array}\right]<0
$$

Where $\Pi_{i}=A_{i}^{T} L_{i}+L_{i}^{T} A_{i}-A_{i}^{T} R^{T} \Phi R A_{i}-E^{T} P_{i} E$.

Lemma 2: The discrete-time singular Markovian jump systems eq. (1) with $\Delta A\left(r_{k}\right), \Delta B\left(r_{k}\right), u(k)=0$ and partially unknown transition probabilities are stochastically admissible and if and only if there exist a set of positive definite matrices $P_{i}, \quad i \in l$, a symmetric and nonsingular matrix $\Phi$, satisfying

$$
A_{i}^{T}\left(\sum_{j=1}^{N} \pi_{i, j} \bar{P}_{j}-R^{T} \Phi R\right) A_{i}-E^{T} P_{i} E<0,
$$

Where $\bar{P}_{i}=\sum_{j_{1} \in l_{k}^{i}} \pi_{i, j_{1}} P_{j_{1}}+h_{i} P_{j_{2}}$,

$$
h_{i}=\Sigma_{j_{2} \in l_{u k}^{i}} \pi_{i, j_{2}}=1-\Sigma_{j_{1} \in l_{k}^{i}} \pi_{i, j_{1}},
$$

Lemma 3: Let $G$ be a real symmetric matrix and $D, H$ Be real matrices with appropriate dimensions. Then, $G+D F(k) H+(D F(k) H)^{T}<0$ holds for any $F^{T}(k) F(k) \leq I$, if and only if there exist a constant sclar $\varepsilon>0$ satisfying $G+\varepsilon D D^{T}+\varepsilon^{-1} H^{T} H<0$.

Remark 1: For each $G_{i}$ are of full row rank, and $C_{i} C_{i}^{\perp}=0$, the invertible matrices $T_{i}$ generally are not unique. $A$ special $T_{i}$ can be obtained by

$$
T_{i}=\left[\begin{array}{ll}
C_{i}^{T}\left(C_{i} C_{i}^{T}\right)^{-1} & C_{i}^{\perp}
\end{array}\right]
$$

Then, we have

Consider the following static output feedback controller

$$
u(k)=K\left(r_{k}\right) y(k)
$$

Where $K\left(r_{k}\right)$ are the static output feedback gain to be

Determined. Substituting eq. (4) into system eq. (1) yields the closed-loop systems:

$$
\operatorname{Ex}(k+1)=\left(A_{i}+\Delta A_{i}+\left(B_{i}+\Delta B_{i}\right) K_{i} C_{i}\right) x(k),
$$

Theorem 1: The discrete-time singular Markovian jump systems eq.(7) with partially unknown transition probabilities are stochastically admissible if and only if there exist a set of positive definite matrices $P_{i}, i \in l$, a symmetric and nonsingular matrix $\Phi$, satisfying

$$
\bar{A}_{i}^{T}\left(\bar{P}_{i}-R^{T} \Phi R\right) \bar{A}_{i}-T_{i}^{T} E^{T} P_{i} E T_{i}<0,
$$

Where

$$
\begin{gathered}
\bar{A}_{i}=\left(A_{i}+\Delta A_{i}+\left(B_{i}+\Delta B_{i}\right) K_{i} C_{i}\right), \\
h_{i}=\sum_{j_{2} \in \in_{u k}^{i}} \pi_{i, j_{2}}=1-\Sigma_{j_{1} \in l_{k}^{i}} \pi_{i, j_{1}}, \\
\bar{P}_{i}=\sum_{j_{1} \in l_{k}^{i}} \pi_{i, j_{1}} P_{j_{1}}+h_{i} P_{j_{2}} .
\end{gathered}
$$

Theorem 2: For each $i \in l$, let $W_{1}, W_{2}$ be the given appropriate matrices, the corresponding closed-loop systems eq.(7) with partially unknown transition probabilities are regular, causal and stochastically stable if there exists positive definite matrices $Y_{i}, \Psi$, nonsingular matrices $G_{i}$ and $H_{i}$ satisfying the following matrix inequalities

$$
\left(\begin{array}{ccc}
\bar{\Omega}_{i} & \Lambda_{i}^{T} & 0 \\
* & -G_{i}-G_{i}^{T} & M_{i}^{T} \\
* & * & -\Theta_{i}
\end{array}\right)<0,
$$

Where

$$
\begin{aligned}
\bar{\Omega}_{i}= & G_{i}^{T}\left(\left(A_{i}+\Delta A_{i}\right) T_{i}\right)^{T}+\left(A_{i}+\Delta A_{i}\right) T_{i} G_{i} \\
& +\left(\left(B_{i}+\Delta B_{i}\right) Z_{i}\right)^{T}+\left(B_{i}+\Delta B_{i}\right) Z_{i} \\
& -W_{1}^{T} E G_{i}-G_{i}^{T} E^{T} W_{1}-W_{2}^{T} R\left(A_{i}+\Delta A_{i}\right) T_{i} G_{i} \\
& -G_{i}^{T} T\left(A_{i}+\Delta A_{i}\right)^{T} R^{T} W_{2}-W_{2}^{T} R\left(B_{i}+\Delta B_{i}\right) \\
& Z\left(\left(B_{i}+\Delta B_{i}\right) Z\right)^{T} R^{T} W_{2}+W_{1}^{T} Y_{i} W_{1}+W_{2}^{T} \Psi W_{2} \\
\Lambda_{i} & =\left(A_{i}+\Delta A_{i}\right) T_{i} G_{i}+\left(B_{i}+\Delta B_{i}\right) Z_{i}-G_{i}^{T}, \\
M_{i}= & {\left[\sqrt{\pi_{i 1}} G_{i}^{T}, \sqrt{\pi_{i 2}} G_{i}^{T}, \ldots, \sqrt{\pi_{i j_{1}}} G_{i}^{T}, \sqrt{h} G_{i}^{T}\right]^{T}, }
\end{aligned}
$$




$$
\begin{gathered}
\Theta_{i}=\operatorname{diag}\left[Y_{1}, Y_{2}, \ldots, Y_{j_{1}}, Y_{j_{2}}\right], \\
Z_{i}=\left[\begin{array}{ll}
H_{i} & 0
\end{array}\right], \quad H_{i}=K_{i} G_{1 i} .
\end{gathered}
$$

Proof. By applying Lemma 1 to Theorem 1 for each $i \in l$, it Follows that inequalities (8) holds if

$$
\left[\begin{array}{cc}
\bar{\Omega}_{i} & \bar{A}_{i} L_{i}-L_{i}^{T} \\
L_{i}^{T} \bar{A}_{i}-L_{i} & \bar{P}_{i}-L_{i}-L_{i}^{T}
\end{array}\right]<0,
$$

Where

$$
\begin{gathered}
\bar{A}_{i}=\left(A_{i}+\Delta A_{i}+\left(B_{i}+\Delta B_{i}\right) K_{i} C_{i}\right), \\
\bar{\Omega}_{i}=\bar{G}_{i}^{T} \bar{A}_{i}^{T}+\bar{A}_{i} G_{i}-W_{i}^{T} E G_{i}-G_{i}^{T} E^{T} W_{1}-W_{2}^{T} R \bar{A}_{i} G_{i} \\
-G_{i}^{T} \bar{A}_{i}^{T} R^{T} W_{2}+W_{1}^{T} Y_{i} W_{1}+W_{2}^{T} \Psi W_{2},
\end{gathered}
$$

Let $L_{i}^{-1}=G_{i}$ and pre- and post-multiplying eq. (10) by both $\bar{G}_{i}=\operatorname{diag}\left[\begin{array}{ll}G_{i} & G_{i}\end{array}\right]^{T}$ and its transpose, then we have

$$
\left[\begin{array}{cc}
\Upsilon_{i} & G_{i}^{T} \bar{A}_{i}^{T}-G_{i} \\
* & G_{i}^{T}\left(\bar{P}_{k i}+h_{i} P_{j_{2}}\right) G_{i}-G_{i}-G_{i}^{T}
\end{array}\right]<0
$$

Where

$\Upsilon_{i}=G_{i}^{T} \bar{A}_{i}^{T}+\bar{A}_{i} G_{i}-G_{i}^{T} \bar{A}_{i}^{T} R^{T} \Psi^{-1} R \bar{A}_{i} G_{i}-G_{i}^{T} E^{T} P_{i} E G_{i}$,

$\bar{P}_{k i}=\sum_{j_{1} \in l_{k}^{i}} \pi_{i, j_{1}} P_{j_{1}}$,

$\bar{A}_{i}=\left(A_{i}+\Delta A_{i}+\left(B_{i}+\Delta B_{i}\right) K_{i} C_{i}\right)$,

Let $Y_{i}^{-1}=P_{i}$, by using the Schur complement lemma, it is easy to show that

$$
\left[\begin{array}{ccc}
\Gamma_{i} & G_{i}^{T} \bar{A}_{i}^{T}-G_{i} & 0 \\
* & -G_{i}-G_{i}^{T} & M_{i}^{T} \\
* & * & -\Theta_{i}
\end{array}\right]<0
$$

Where

$\Gamma_{i}=G_{i}^{T} \bar{A}_{i}^{T}+\bar{A}_{i} G_{i}-G_{i}^{T} \bar{A}_{i}^{T} R^{T} \Psi^{-1} R \bar{A}_{i} G_{i}-G_{i}^{T} E^{T} Y_{i}^{-1} E G_{i}$,

$M_{i}=\left[\sqrt{\pi_{i 1}} G_{i}^{T}, \sqrt{\pi_{i 2}} G_{i}^{T}, \ldots, \sqrt{\pi_{i j_{1}}} G_{i}^{T}, \sqrt{h} G_{i}^{T}\right]^{T}$,

$\Theta_{i}=\operatorname{diag}\left[Y_{1}, Y_{2}, \ldots, Y_{j_{1}}, Y_{j_{2}}\right]$

$\bar{A}_{i}=\left(A_{i}+\Delta A_{i}+\left(B_{i}+\Delta B_{i}\right) K_{i} C_{i}\right)$.

According to Lemma 3, choose the appropriate matrices $W_{1}, W_{2}$, such that

$$
\begin{aligned}
0 \leq\left(G_{i}^{T}\right. & \left.E^{T}-W_{1}^{T} Y_{i}\right) Y_{i}^{-1}\left(E G_{i}-Y_{i} E_{1}\right) \\
=G_{i}^{T} & E^{T} Y_{i}^{-1} E G_{i}-W_{1}^{T} E G_{i}-G_{i}^{T} E^{T} W_{1}+W_{1}^{T} Y_{i} W_{1} \\
0 \leq & \left(G_{i}^{T} \bar{A}_{i}^{T} R^{T}-W_{2}^{T} \Psi\right) \Psi^{-1}\left(R \bar{A}_{i} G_{i}-\Psi W_{2}\right) \\
= & G_{i}^{T} \bar{A}_{i}^{T} R^{T} \Psi^{-1} R \bar{A}_{i} G_{i}-W_{2}^{T} R \bar{A}_{i} G_{i} \\
& -G_{i}^{T} \bar{A}_{i}^{T} R^{T} W_{2}+W_{2}^{T} \Psi W_{2}
\end{aligned}
$$

It is easy to show that (13) and (14) can be rewritten as

$$
-G_{i}^{T} E^{T} Y_{i}^{-1} E G_{i} \leq-W_{1}^{T} E G_{i}-G_{i}^{T} E^{T} W_{1}+W_{1}^{T} Y_{i} W_{1}
$$

$-G_{i}^{T} \bar{A}_{i}^{T} R^{T} \Psi^{-1} R \bar{A}_{i} G_{i} \leq-W_{2}^{T} R \bar{A}_{i} G_{i}-G_{i}^{T} \bar{A}_{i}^{T} R^{T} W_{2}+W_{2}^{T} \Psi W_{2}$

From eq. (12), eq. (15) and eq. (16), we have

$$
\left[\begin{array}{ccc}
\Gamma_{i} & G_{i}^{T} \bar{A}_{i}^{T}-G_{i} & 0 \\
* & -G_{i}-G_{i}^{T} & W_{i}^{T} \\
* & * & -\Theta_{i}
\end{array}\right] \leq\left[\begin{array}{ccc}
\Omega_{i} & G_{i}^{T} \bar{A}_{i}^{T}-G_{i} & 0 \\
* & -G_{i}-G_{i}^{T} & W_{i}^{T} \\
* & * & -\Theta_{i}
\end{array}\right]
$$

Where

$$
\begin{aligned}
\Gamma_{i}= & G_{i}^{T} \bar{A}_{i}^{T}+\bar{A}_{i} G_{i}-G_{i}^{T} \bar{A}_{i}^{T} R^{T} \Psi^{-1} R \bar{A}_{i} G_{i}-G_{i}^{T} E^{T} Y_{i}^{-1} E G_{i}, \\
\Omega_{i}= & G_{i}^{T} \bar{A}_{i}^{T}+\bar{A}_{i} G_{i}-W_{1}^{T} E G_{i}-G_{i}^{T} E^{T} W_{1}-W_{2}^{T} R \bar{A}_{i} G_{i} \\
& -G_{i}^{T} \bar{A}_{i}^{T} R^{T} W_{2}+W_{1}^{T} Y_{i} W_{1}+W_{2}^{T} \Psi W_{2}, \\
\bar{A}_{i}= & \left(A_{i}+\Delta A_{i}+\left(B_{i}+\Delta B_{i}\right) K_{i} C_{i}\right) .
\end{aligned}
$$

Considering inequalities above. The inequalities eq. (8) hold if

$$
\left[\begin{array}{ccc}
\Omega_{i} & G_{i}^{T} \bar{A}_{i}^{T}-G_{i} & 0 \\
* & -G_{i}-G_{i}^{T} & W_{i}^{T} \\
* & * & -\Theta_{i}
\end{array}\right]<0
$$

Let matrices $G_{i}$ in the form of $\left[\begin{array}{cc}G_{1 i} & 0 \\ G_{2 i} & G_{31}\end{array}\right]$ andare nonsingular, $G_{2 i} \in R^{(n-q) \times q}$ and $G_{3 i} \in R^{(n-q) \times(n-q)}$ are arbitrary matrices, and let $K_{i} G_{1 i}=H_{i}, Z_{i}=\left[\begin{array}{ll}H_{i} & 0\end{array}\right]$, then we have eq. (9). This completes the proof.

In order to design a static output feedback controller $u(k)=K\left(r_{k}\right) y(k)$ for (1) in the form of LMIs. Theorem 2 will be replaced by the following theorem.

Theorem 3: For each $i \in l$, let $W_{1}, W_{2}$ be the given appropriate matrices, the corresponding closed-loop systems eq.(7) with partially unknown transition probabilities are regular, causal and stochastically stable if there exists positive definite matrices $Y_{i}, \Psi$, nonsingular matrices $G_{i}$ and $H_{i}$ satisfying the following matrix inequalities 


$$
\left[\begin{array}{ccccccccc}
\Gamma_{1 i} & \Gamma_{2 i} & 0 & \Gamma_{4 i} & \Gamma_{5 i} & \Gamma_{6 i} & \Gamma_{7 i} & \Gamma_{8 i} & \Gamma_{9 i} \\
* & \Gamma_{3 i} & M_{i}^{T} & 0 & 0 & 0 & 0 & 0 & 0 \\
* & * & \Theta_{i} & 0 & 0 & 0 & 0 & 0 & 0 \\
* & * & * & \gamma_{1 i} & 0 & 0 & 0 & 0 & 0 \\
* & * & * & * & \gamma_{2 i} & 0 & 0 & 0 & 0 \\
* & * & * & * & * & \gamma_{3 i} & 0 & 0 & 0 \\
* & * & * & * & * & * & \gamma_{4 i} & 0 & 0 \\
* & * & * & * & * & * & * & \gamma_{5 i} & 0 \\
* & * & * & * & * & * & * & * & \gamma_{6 i}
\end{array}\right]<0,
$$

Where

$$
\begin{aligned}
\Gamma_{1 i}= & G_{i}^{T} T_{i}^{T} A_{i}^{T}+A_{i} T_{i} G_{i}+B_{i} Z_{i}+\left(B_{i} Z_{i}\right)^{T} \\
& -W_{1}^{T} E G_{i}-G_{i}^{T} E^{T} W_{1}-W_{2}^{T} R A_{i} T_{i} G_{i}-W_{2}^{T} R B_{i} Z_{i} \\
& -G_{i}^{T} T_{i}^{T} A_{i}^{T} R W_{2}-\left(B_{i} Z_{i}\right)^{T} R^{T} W_{2}+W_{1}^{T} Y_{i} W_{1}+W_{2}^{T} \Psi W_{2} \\
& +\gamma_{1 i} D_{i} D_{i}^{T}+\gamma_{2 i} D_{i} D_{i}^{T}+\gamma_{3 i} W_{2}^{T} R D_{i} D_{i}^{T} R^{T} W_{2}^{T} \\
& +\gamma_{4 i} W_{2}^{T} R D_{i} D_{i}^{T} R^{T} W_{2}^{T}, \\
\Gamma_{2 i}= & G_{i}^{T}\left(A_{i} T_{i}\right)^{T}+\left(B_{i} Z_{i}\right)^{T}-G_{i}, \\
\Gamma_{3 i}= & -G_{i}-G_{i}^{T}+\left(\gamma_{1 i}+\gamma_{2 i}\right) D_{i} D_{i}^{T}, \\
\Gamma_{4 i}= & \Gamma_{6 i}=\Gamma_{8 i}=G_{i}^{T}\left(M_{1 i} T_{i}\right)^{T}, \\
\Gamma_{5 i}= & \Gamma_{7 i}=\Gamma_{9 i}=\left(M_{2 i} Z_{i}\right)^{T}, \\
M_{i}= & {\left[\sqrt{\pi_{i 1}} G_{i}^{T}, \sqrt{\pi_{i 2}} G_{i}^{T}, \ldots, \sqrt{\pi_{i j_{1}}} G_{i}^{T}, \sqrt{h} G_{i}^{T}\right]^{T}, } \\
\Theta_{i}= & \operatorname{diag}\left[Y_{1}, Y_{2}, \ldots, Y_{j_{1}}, Y_{j_{2}}\right], Z_{i}=\left[H_{i} \quad 0\right], H_{i}=K_{i} G_{1 i}, \\
\gamma_{1 i}= & -\beta_{1 i} I, \gamma_{2 i}=-\beta_{2 i} I, \\
\gamma_{3 i}=- & \alpha_{1 i} I, \gamma_{4 i}=-\alpha_{2 i} I, \gamma_{5 i}=-\alpha_{3 i} I, \gamma_{6 i}=-\alpha_{4 i} I .
\end{aligned}
$$

In this case, the gains of the stabilizing static output feedback controller are given by $K_{i}=H_{i} G_{i}^{-1}$.

Proof. Firstly, from Theorem 2, we know that eq. (7) are robustly stochastically admissible if there exist positive definite matrices $Y_{i}, \Psi$, nonsingular matrices $G_{i}$ and $H_{i}$ and the gains of the stabilizing static output feedback controller $K_{i}=H_{i} G_{i}^{-1}$, eq. (9) holds for each $i \in l$.

Next, the matrices of inequalities eq. (9) can be decomposed as

$$
\left(\begin{array}{ccc}
\bar{\Omega}_{i} & \Lambda_{i}^{T} & 0 \\
* & -G_{i}-G_{i}^{T} & M_{i}^{T} \\
* & * & -\Theta_{i}
\end{array}\right)=\left(\begin{array}{ccc}
\bar{\Omega}_{1 i} & G_{i}^{T}\left(A_{i} T_{i}\right)^{T}+\left(B_{i} Z_{i}\right)^{T}-G_{i} & 0 \\
* & -G_{i}-G_{i}^{T} & M_{i}^{T} \\
* & * & -\Theta_{i}
\end{array}\right)
$$

$$
\begin{aligned}
& +\left(\begin{array}{ccc}
\bar{\Omega}_{2 i} & G_{i}^{T}\left(\Delta A_{i} T_{i}\right)^{T}+\left(\Delta B_{i} Z_{i}\right)^{T} & 0 \\
* & 0 & 0 \\
* & * & 0
\end{array}\right) \\
& =\left(\begin{array}{ccc}
\bar{\Omega}_{1 i} & G_{i}^{T}\left(A_{i} T_{i}\right)^{T}+\left(B_{i} Z_{i}\right)^{T}-G_{i} & 0 \\
* & -G_{i}-G_{i}^{T} & M_{i}^{T} \\
* & * & -\Theta_{i}
\end{array}\right) \\
& +\left(\begin{array}{ccc}
\bar{\Omega}_{2 i} & 0 & 0 \\
0 & 0 & 0 \\
0 & 0 & 0
\end{array}\right)+\left(\begin{array}{ccc}
0 & G_{i}^{T}\left(\Delta A_{i} T_{i}\right)^{T}+\left(\Delta B_{i} Z_{i}\right)^{T} & 0 \\
* & 0 & 0 \\
0 & 0 & 0
\end{array}\right) \\
& =\left(\begin{array}{ccc}
\bar{\Omega}_{1 i} & G_{i}^{T}\left(A_{i} T_{i}\right)^{T}+\left(B_{i} Z_{i}\right)^{T}-G_{i} & 0 \\
* & -G_{i}-G_{i}^{T} & M_{i}^{T} \\
* & * & -\Theta_{i}
\end{array}\right) \\
& +\left(\begin{array}{ccc}
\bar{\Omega}_{2 i} & 0 & 0 \\
0 & 0 & 0 \\
0 & 0 & 0
\end{array}\right)+\left[\begin{array}{ccc}
0 & G_{i}^{T}\left(D_{i} F(k)_{i} M_{1 i} T_{i}\right)^{T} & 0 \\
* & 0 & 0 \\
0 & 0 & 0
\end{array}\right] \\
& +\left[\begin{array}{ccc}
0 & \left(D_{i} F(k)_{i} M_{2 i} Z_{i}\right)^{T} & 0 \\
* & 0 & 0 \\
0 & 0 & 0
\end{array}\right]<0
\end{aligned}
$$

Where

$$
\begin{aligned}
\bar{\Omega}_{1 i}= & G_{i}^{T} T_{i}^{T} A_{i}^{T}+A_{i} T_{i} G_{i}+B_{i} Z_{i}+\left(B_{i} Z_{i}\right)^{T} \\
& -W_{1}^{T} E G_{i}-G_{i}^{T} E^{T} W_{1}-W_{2}^{T} R A_{i} T_{i} G_{i}-W_{2}^{T} R B_{i} Z_{i} \\
& -G_{i}^{T} T_{i}^{T} A_{i}^{T} R^{T} W_{2}-\left(B_{i} Z_{i}\right)^{T} R^{T} W_{2}+W_{1}^{T} Y_{i} W_{1}+W_{2}^{T} \Psi W_{2},
\end{aligned}
$$

According to Lemma 3, eq. (9) hold if there exist scalars $\alpha_{1 i}>0, \alpha_{2 i}>0, \alpha_{3 i}>0, \alpha_{4 i}>0, \beta_{1 i}>0, \beta_{2 i}>0$ for each $i \in l$, such that

$$
\begin{aligned}
& \left(\begin{array}{ccc}
\bar{\Omega}_{1 i} & G_{i}^{T}\left(A_{i} T_{i}\right)^{T}+\left(B_{i} Z_{i}\right)^{T}-G_{i} & 0 \\
* & -G_{i}-G_{i}^{T} & M_{i}^{T} \\
* & * & -\Theta_{i}
\end{array}\right) \\
& +\left[\begin{array}{ccc}
\hat{\Omega}_{2 i} & 0 & 0 \\
0 & 0 & 0 \\
0 & 0 & 0
\end{array}\right]+\left[\begin{array}{ccc}
0 & 0 & 0 \\
0 & \left(\beta_{1 i}+\beta_{2 i}\right) D_{i} D_{i}^{T} & 0 \\
0 & 0 & 0
\end{array}\right]
\end{aligned}
$$

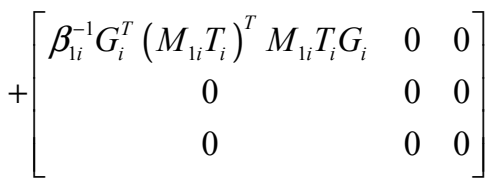




$$
+\left[\begin{array}{ccc}
\beta_{2 i}^{-1}\left(M_{2 i} Z_{i}\right)^{T} M_{2 i} Z_{i} & 0 & 0 \\
0 & 0 & 0 \\
0 & 0 & 0
\end{array}\right]<0
$$

Where

$$
\begin{aligned}
\hat{\Omega}_{2 i}= & \alpha_{1 i} D_{i} D_{i}^{T}+\alpha_{2 i} D_{i} D_{i}^{T}+\alpha_{3 i} W_{2}^{T} R D_{i} D_{i}^{T} R^{T} W_{2}^{T} \\
& +\alpha_{4 i} W_{2}^{T} R D_{i} D_{i}^{T} R^{T} W_{2}^{T}+\alpha_{1 i}^{-1} G_{i}^{T}\left(M_{1 i} T_{i}\right)^{T} M_{1 i} T_{i} G_{i} \\
& +\alpha_{2 i}^{-1}\left(M_{2 i} T_{i}\right)^{T} M_{2 i} Z_{i}+\alpha_{3 i}^{-1} G_{i}^{T}\left(M_{1 i} T_{i}\right)^{T} M_{1 i} T_{i} G_{i} \\
& +\alpha_{4 i}^{-1}\left(M_{2 i} T_{i}\right)^{T} M_{2 i} Z
\end{aligned}
$$

(18).

By using the Schur complement lemma, we have LMIs eq.

This proof can be completed.

Remark 2: If $l_{u k}^{i}=\varnothing$, then $\sum_{j_{1} \in l_{k}^{i}} \pi_{i, j_{1}}=1$ for every $i \in l$.

It means that the elements in every $i$ th row are all known.

$$
h_{i}=\sum_{j_{2} \in l_{u k}^{i k}} \pi_{i, j_{2}}=1-\sum_{j_{1} \in l_{k}^{i}} \pi_{i, j_{1}}=0
$$

Moreover, the transition probabilities with partially unknown or completely known, which can still be viewed as accessible in the sense of this paper. Therefore, our transition probabilities matrix considered in the sequel is a more natural assumption to the singular Markovian jump systems and hence convers the existing ones.

Remark 3: From the proof of Theorem 2, it is easy to see that matrices $W_{1}$ and $W_{2}$ can be arbitrary. So the matrix inequalities in eq. (9) can be viewed as a standard LMI when matrices $W_{1}$ and. Define two scalars $\delta$ and $\eta$ satisfying:

$$
\min _{\delta}\left\|E G_{i}-Y_{i} W_{1}\right\|_{1} \leq \delta, \min _{\eta}\left\|R A_{i} G_{i}-\Psi W_{2}\right\|_{1} \leq \eta
$$

s.t. eq. (9). We have pointed out that in order to fix the matrices $W_{1}$ and $W_{2}$, a matrix equality constant has to be involved, which forms a minimization problem.

Based on the earlier discussion, the following algorithm is to be presented

Iterative LMI Algorithm:

Step 1: For desired decay rate $\delta \geq 0$ and $\eta \geq 0$ give the initial matrices $W_{1}$ and $W_{2}$, and find a feasible solution for the linear matrix inequalities eq. (9). Denote the feasible solution as $\left(\delta_{0}, \eta_{0}, G_{i 0}, Y_{i 0}, \Psi_{0}\right)$. Take $G_{i 0}, Y_{0 i}$ and $\Psi_{0}$ as the iterative initial values.

Step 2: Given the initial values $\left(\delta_{0}, \eta_{0}, G_{i 0}, Y_{i 0}, \Psi_{0}\right)$, solve the minimization problem:

$$
\begin{aligned}
& \min _{\delta}\left\|E G_{i 0}-Y_{i 0} W_{11}\right\| \leq \delta_{0}, \\
& \min _{\eta}\left\|R A_{i} G_{i 0}-\Psi_{0} W_{21}\right\|_{1} \leq \eta_{0}
\end{aligned}
$$

Denote the minimizing solution as $\left(W_{11}, W_{21}\right)$.
Step 3: If $\delta_{1} \geq \delta_{0}, \eta_{1} \geq \eta_{0}$. Then, stop. Otherwise, go to step 2.

Remark 4: In Theorem 2, appropriate matrices $W_{1}$ and can guarantee the matrices $\left(G_{i}^{T} E^{T}-W_{1}^{T} Y_{i}\right) Y_{i}^{-1}\left(E G_{i}-Y_{i} W_{1}\right)$ and $\left(G_{i}^{T} A_{i}^{T} R^{T}-W_{2}^{T} \Psi\right) \Psi^{-1}\left(R A_{i} G_{i}-\Psi W_{2}\right)$ in eq. (13) and eq. (14) tend to zero, which has reduced the conservatism. It is not only easy to obtain the solutions of eq. (9) and the matrix inequalities of the following theorem, but also to reduce the conservatism compared with Theorem 13 in [11], which has used two scalars. Especially, when we choose $W_{1}$ and $W_{2}$ in terms of $W_{1}=\operatorname{diag}\left[\alpha_{1}, \alpha_{1}, \ldots, \alpha_{1}\right]$ and $W_{2}=\operatorname{diag}\left[\alpha_{2}, \alpha_{2}, \ldots, \alpha_{2}\right]$, it can be seen that matrix parameters in handing this problem by applying a set of matrix operations.

Remark 5: It is noted that Theorem 2 are degenerated to Theorem 12 in [11], when we choose $W_{1}$ and $W_{2}$ in terms of $W_{1}=\operatorname{diag}\left[\alpha_{1}, \alpha_{1}, \ldots, \alpha_{1}\right]$ and $W_{2}=\operatorname{diag}\left[\alpha_{2}, \alpha_{2}, \ldots, \alpha_{2}\right]$.

\section{Numerical Examples and Simulation}

In this section, some numerical examples will be given to show the validity of the develop theoretical results.

\section{Example 1:}

Consider the discrete-time uncertain singular Markovian Jump systems eq. (1) with the following parameters:

$$
\begin{aligned}
& E=\left[\begin{array}{cc}
4.1 & 0 \\
0 & 0
\end{array}\right], A_{1}=\left[\begin{array}{cc}
4.9 & 0 \\
1 & 3.6
\end{array}\right], \quad A_{2}=\left[\begin{array}{cc}
4.1 & 0.3 \\
0 & 3.1
\end{array}\right], \\
& B_{1}=\left[\begin{array}{cc}
2.2 & -0.8 \\
0 & 0.5
\end{array}\right], \quad B_{2}=\left[\begin{array}{cc}
0.8 & 1 \\
-0.6 & 2.5
\end{array}\right], \\
& C_{1}=\left[\begin{array}{ll}
1 & -0.1
\end{array}\right], \quad C_{2}=\left[\begin{array}{ll}
1 & 0.1
\end{array}\right], \quad R=\left[\begin{array}{cc}
0 & 0 \\
0 & 1.5
\end{array}\right], \\
& M_{11}=\left[\begin{array}{ll}
0.2 & 0
\end{array}\right], \quad M_{12}=\left[\begin{array}{ll}
0.1 & 0
\end{array}\right], \\
& M_{21}=\left[\begin{array}{ll}
0.2 & 1
\end{array}\right], \quad M_{22}=\left[\begin{array}{ll}
0.1 & 1
\end{array}\right], \\
& T_{1}=\left[\begin{array}{cc}
0.9901 & 0.0995 \\
-0.099 & 0.995
\end{array}\right], \quad T_{2}=\left[\begin{array}{cc}
0.9901 & -0.0995 \\
0.099 & 0.995
\end{array}\right],
\end{aligned}
$$

The transition probability matrix of form is given by.

$$
\pi=\left[\begin{array}{cc}
0.5 & 0.5 \\
? & ?
\end{array}\right],
$$

Our goal is to design a static output feedback controllers such that the closed-loop systems are stochastically stable. According to Theorem 3 and Remark 3, let

$W_{1}=\left[\begin{array}{cc}1.5 & 0.1 \\ 0 & 1\end{array}\right], \quad W_{2}=\left[\begin{array}{cc}2 & 0.1 \\ 0 & 2\end{array}\right]$,

Designed for control the gains of the stabilizing static output feedback controller: 
$K_{1}=\left[\begin{array}{c}-1.7302 \\ 0.0691\end{array}\right], \quad K_{2}=\left[\begin{array}{l}-2.7815 \\ -0.6312\end{array}\right]$,

After applying Theorem 3, trajectory simulation for the closed-loop system systems shown in Fig. 1 are stochastically admissible with the same Markovian jump process under the given initial condition $x_{0}=[0.1,-0.9]^{T}$.

Example 2:

Consider the discrete-time uncertain singular Markovian Jump Systems eq. (1) with the parameters the same as in Example 1 except

$A_{1}=\left[\begin{array}{cc}4.9 & 0 \\ 1 & 3.6\end{array}\right], \quad A_{2}=\left[\begin{array}{cc}4.1 & 0.3 \\ 0 & 3.1\end{array}\right]$,

$M_{11}=M_{12}=M_{21}=M_{22}=\left[\begin{array}{ll}0 & 0\end{array}\right]$,

The transition probability matrix of form is given by eq. (19). The switching of the mode used in the simulation is shown in Fig. 2. Our goal is to design a controller eq. (7) are stochastically stable. According to Theorem 2 and Remark 3, let

$W_{1}=\left[\begin{array}{cc}1.5 & 0.1 \\ 0 & 1\end{array}\right], \quad W_{2}=\left[\begin{array}{cc}2 & 0.1 \\ 0 & 2\end{array}\right]$,

Designed for control the gains of the stabilizing static output feedback controller:

$K_{1}=[-6.3933], \quad K_{2}=[-6.2710]$.

Applying this controller makes the closed-loop systems eq. (7) stochastically stable. Fig. 4 shows that the closed-loop systems trajectories of the given initial condition $x_{0}=[1,-0.9]^{T}$ tend to be the zero equilibrium. That is to say, this number example is finally stochastically admissible.

Remark 6: Fig. 4 shows the admissibility analysis Theorem 2 to solve the output feedback controller with two matrices

$W_{1}=\left[\begin{array}{cc}1.5 & 0.1 \\ 0 & 1\end{array}\right], \quad W_{2}=\left[\begin{array}{cc}2 & 0.1 \\ 0 & 2\end{array}\right]$,

(o) and two scalars $W_{1}=1$ and $W_{2}=2(+)$, when the transition probabilities of the systems are partially unknown. It is shown that the solution of the matrix inequalities, which use matrices in Theorem 2 can be obtained easier.

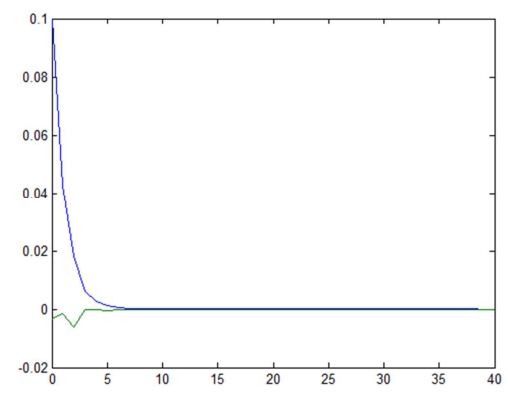

Figure 1. State response of the closed-loop systems eq. (7) for the robust static output feedback control.

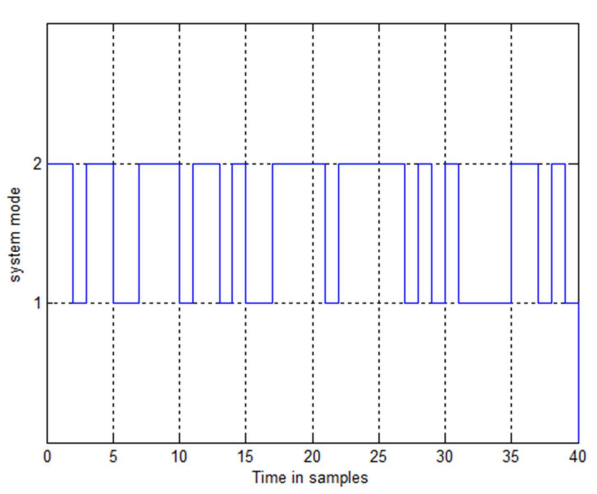

Figure 2. Mode evolution $r_{k}$.

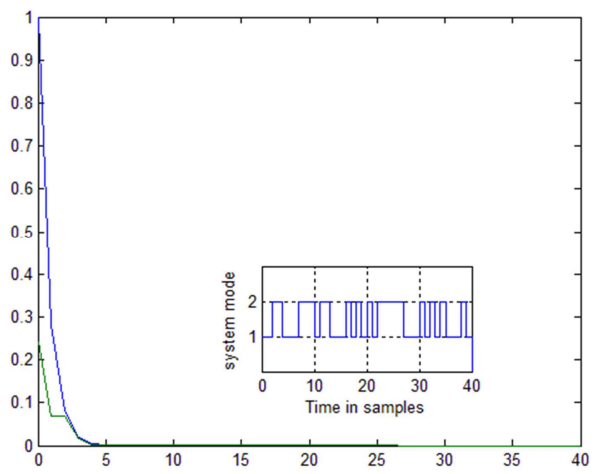

Figure 3. State response of the closed-loop systems eq. (7) for static output feedback control.

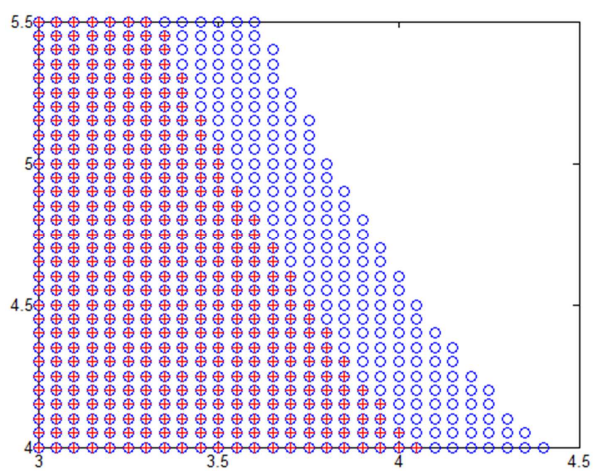

Figure 4. Admissibility analysis with Theorem 2 use two matrices (o) and two scalars (+).

\section{Conclusion}

In this paper, we deal with the problem of the static output feedback control problem for a class of discrete-time singular Marovian jump systems with partly unknown transition rates. The considered systems are more general than the systems with completely known transition rates or completely unknown transition probabilities. In terms of linear matrix inequalities, based on a necessary and sufficient condition of the stochastic stability with partially unknown transition probabilities of the unforced systems, some sufficient conditions are obtained to design of a static output feedback controller and a robust static output feedback controller, which 
guarantee that the closed-loop systems are piecewise regular, causal and stochastically stable by employing the linear matrix inequality technique. Some numerical examples have shown the validity and the applicability of the developed results. The future work will focus on the study of discrete-time Markovian jump singularly perturbed systems.

\section{References}

[1] P. Bikash, C. Balarko, Robust Control in Power Systems, Berlin, Springer, 2005.

[2] T. Kaczorek, "Singular fractional linear systems and electrical circuits", International Journal of Applied Mathematics and Computer Science, vol. 21, no. 2, pp. 379-384, Jun 2011.

[3] J. Zhang, Control of Generalized Economic Systems, Beijing, Tsinghua Press, 1990.

[4] Q. Zhang, C. Liu, X. Zhang, Complexity, analysis and control of singular biological systems, London.

[5] L. Dai, Singular Control Systems, New York, Springer, 1989. (2004) 568-574.

[6] S. Xu, J. Lam, Robust Control and Filtering of Singular Systems, Berlin, Springer, 2006.

[7] S. Xu, B. Song, J. Lu, J. Lam, "Robust stability of uncertain discrete-time singular fuzzy systems", Fuzzy Sets and Systems, vol. 158, no. 20, pp. 2306-2316, October 2007.

[8] S. Xu, J. Lam, C. Yang, "Robust $\mathrm{H} \propto$ control for discrete singular systems with state delay and parameter uncertainty", dynamics of continuous. Discrete And Impulsive Systems SeriesB: Application And Al-gorithm, vol. 9, no. 4, pp. 539-554, 2002.

[9] S. Ma, C. Zhang, Z. Cheng, "Delay-dependent robust $\mathrm{H} \infty$ control for uncertain discrete-time singular systems with time-delay", Journal of Computational and Applied Mathematics, vol. 217, no. 1, pp. 194-211, July 2008.

[10] A. Trofino-Neto, V. Kucera, "Stabilization via static output feedback", IEEE Trans. Autom. Control, vol. 38, no. 5, pp. 764-765, May 1993.

[11] Y. Cao, J. Lam, "Stochastic stabilizability and $\mathrm{H} \infty$ control for discrete-time jump linear systems with time delay", Journal of the Franklin Institute, vol. 336, no. 8, pp. 1263-1281, November 1999.

[12] Y. Cao, J. Lam, "Robust $\mathrm{H} \infty$ control of uncertain Markovian jump systems with time-delay", IEEE Trans. Autom. Control, vol. 45 , no. 1, pp. 77-83, January 2000.

[13] W. Chen, Z. Guan, P. Yu, "Delay-dependent stability and H $\infty$ control of uncertain discrete-time Markovian jump systems with mode-dependent time delays", Systems \& Control Letters, vol. 52, no. 5, pp. 361-376, August 2004.

[14] F. Weng, W. Mao, "Delay-rang-dependent and delay-distribution-independent stability criteria for discrete-time singular Markovian jump systems", International Journal of Control, Automation and Systems, vol. 11, no. 2, pp. 233-242, April 2013.
[15] P. Shi, E. K. Boukas, K. Agarwal, "Control of Markovian jump discrete-time systems with norm bounded uncertainty and unknown delay", IEEE Trans. Autom. Control, vol. 44, no. 11, pp. 2139-2144, 1999.

[16] M. Karan, P. Shi, C. Kaya, "Transition probability bounds for the stochastic stability robustness of continuous- and discrete-time Markovian jump linear systems", Automatica, vol. 42, no. 12, pp. 2159-2168, December 2006.

[17] P. Shi, E. K. Boukas, K. Agarwal, "Kalman filtering for continuous-time uncertain systems with Markovian jumping parameters", IEEE Trans. Automat. Control, vol. 44, no. 8, pp. 1592-1597, 1999.

[18] S. Xu, T. Chen, J. Lam, "Robust $\mathrm{H} \infty$ filtering for uncertain Markovian jump systems with mode-dependent time-delays", IEEE Trans. Automat. Control, vol. 48, no. 5, pp. 900-907, 2003.

[19] J. Xiong, J. Lam, H. Gao, W. Daniel, "On robust stabilization of Markovian jump systems with uncertain switching probabilities", Automatica, vol. 41 no. 5, p-p. G. Wang, "Robust Stabilization of Singular Markovian Jump Systems with Uncertain Switching", International Journal of Control, Automation, and Systems, vol. 11, no. 1, pp. 188-193, February 2013.897-903, May 2005.

[20] X. Zhao, Q. Zeng, "Delay-dependent $\mathrm{H} \infty$ performance analysis for Markovian jump systems with mode-dependent time varying delays and partially known transition rates", International Journal of Control, Automation, and Systems, vol. 8, no. 2, pp. 482-489, April 2010.

[21] X. Yao, L. Guo, "Disturbance attenuation and rejection for discrete-time Markovian jump systems with lossy measurements", Information Sciences, vol. 278, pp. 673-684, September 2014.

[22] Y. Zhang, P. Shi, S. K. Nguang, J. Zhang, H. R. Karimi, "Finite-time boundedness for uncertain discrete neural networks with time-delays and Markovian jumps", Neurocomputing, vol. 140, pp. 1-7, September 2014.

[23] L. Zhang, E. K. Boukas, "Stability and stabilization of Markovian jump linear systems with partly unknown transition probabilities", Automatica, vol. 45, no. 2, pp. 463-468, February 2009.

[24] G. Wang, Q. Zhang, V. Sreeram, "Partially mode-dependent H $\infty$ filtering for discrete-time Markovian jump systems with partly unknown transition probabilities", SignalProcessing, vol. 90, no. 2, 548-556, February 2010.

[25] L. Zhang, J. Lam, "Necessary and Sufficient Conditions for Analysis and Synthesis of Markov Jump Linear Systems With Incomplete Transition Descriptions". IEEE Trans, Automat. Control, vol. 55, no. 7, pp. 1695-1701, 2010.

[26] N. K. Kwon, B. Y. Park, P. G. Park, Less conservative stabilization conditions for Markovian jump systems with incomplete knowledge of transition probabilities and input saturation [J]. Optimal Control Applications and Methods, 2016.

[27] Y. G. Kao, C. H. Wang, New delay-dependent stability of Markovian jump neutral stochastic systems with general unknown transition rates [J]. International Journal of Systems Science, 47 (11): 2499-2509, 2016. 